\title{
Multidimensional Wavelets for Scalable Image Decomposition: Orbital Wavelets
}

\author{
H. M. de Oliveira* $\quad$ V. V. Vermehren ${ }^{\dagger} \quad$ R. J. Cintra ${ }^{\ddagger}$
}

\begin{abstract}
Wavelets are closely related to the Schrödinger's wave functions and the interpretation of Born. Similarly to the appearance of atomic orbital, it is proposed to combine anti-symmetric wavelets into orbital wavelets. The proposed approach allows the increase of the dimension of wavelets through this process. New orbital 2D-wavelets are introduced for the decomposition of still images, showing that it is possible to perform an analysis simultaneous in two distinct scales. An example of such an image analysis is shown.
\end{abstract}

\section{Keywords}

2D wavelets; anti-symmetric wavelets; orthogonal wavelets; image analysis.

\section{Introduction}

Wavelet transform methods are important tools in image processing due to their capabilities for multiresolution analysis and image decomposition [8,25. Wavelet-based image processing find application in computer graphics [36, including radiosity, global illumination [39], and real volume data [28, and volume rendering [33; being routinely considered as an approach for texture image decomposition, image coding, subband coding, fast image segmentation [1, 3, 20, 23, 44, and 3D signal processing [42. In particular, wavelet transform coding [7,12, 30, 43, has emerged as a practical and fully-established tool [3, 25] which benefits image compressing methods, such as the JPEG 2000 standard [15, 35, 37, 43], and multimedia schemes on Internet, such as data streaming over IP [4,31] and image querying [17. Scalable coding for image, audio, and video largely adopts wavelet-based methods as demonstrated in the MPEG-4 codec 7, $7,11,30,31,38$.

Multiwavelets have been explored for the assessment of order/disorder in reconstructed biomedical images [45]. Further connections between information-theoretical entropy and wavelet analysis were explored in the context of geoscience [29]. Recent advances in the field of image decomposition include the proposition of special filters for spherical harmonics modeling [18, capable of multi-level decomposition suitable for 3D images with the introduction of the concept of spherical harmonics entropy [19].

In the same vein of exploring connections between different research fields, Ashmead reported [2] a link between quantum mechanics and wavelets. Despite the potentially deep mathematical meaning of such link, it has not been significantly explored in the context of wavelet decomposition and image analysis. Indeed, the wave nature of light can be deduced from the phenomenon of interference, the photoelectric effect, however, it seems to suggest a corpuscular nature of light. Theoretical physicists struggled to include observations like the photoelectric effect and the wave-particle duality into their formulations [1]. Erwin Schrödinger employed advanced mechanics to address such phenomena and developed an equation that relates the space-time in quantum mechanics. Because wavelets are localized in both time and frequency they offer significant advantages for the analysis of problems in quantum mechanics.

In this paper, we aim at proposing an alternative wavelet decomposition scheme. For such, we explore the above discussed link between wavelets and quantum mechanics and shed some light on some of these relations. Rather than

\footnotetext{
*Departamento de Estatística, Universidade Federal de Pernambuco, Recife, PE, Brazil. E-mail: hmo@de.ufpe.br

${ }^{\dagger}$ Departamento de Engenharia Elétrica, Universidade do Estado do Amazonas, Manaus, AM, Brazil

${ }_{\ddagger}^{\ddagger}$ Departamento de Estatística, Universidade Federal de Pernambuco, Recife, PE, Brazil.
} 
seeking at wavelet features on particles or waves, we adapted some concepts of quantum mechanics to a novel wavelet decomposition for still images.

The paper is organized as follows. Section 2 details the main ideas behind the proposed wavelet system which is based on an interpretation from particle physics and quantum states [5] 14. We describe the construction of the proposed orbital wavelets for the image decomposition. It is shown that the introduced derivation is naturally suitable for generating two-dimensional wavelets. In Section 3 we submit standard imagery to the proposed wavelet decomposition scheme and compare the results with standard wavelet decomposition.. Section 4 concludes the paper.

\section{Orbital Wavelet Decomposition}

\subsection{Particle Systems}

The wave functions describing electronic orbitals can be combined generating atomic orbitals. Let us consider a two-particle non-interaction systems with particles located at position vectors $\mathbf{r}_{1}$ and $\mathbf{r}_{2}$, respectively, where each particle is equipped with wave functions $\psi_{\alpha}\left(\mathbf{r}_{1}\right)$ and $\psi_{\beta}\left(\mathbf{r}_{2}\right)$ at states $\alpha$ and $\beta$, respectively. The wave function that characterizes the orbital interaction of the two particles is furnished by a combination $\psi_{\alpha}\left(\mathbf{r}_{1}\right)$ and $\psi_{\beta}\left(\mathbf{r}_{2}\right)$ in two different configurations: symmetric $(S)$ and anti-symmetric $(A)$ combinations [13. Such combinations are given by:

$$
\begin{aligned}
& \psi_{S}\left(\mathbf{r}_{1}, \mathbf{r}_{2}\right)=\frac{1}{\sqrt{2}}\left[\psi_{\alpha}\left(\mathbf{r}_{1}\right) \cdot \psi_{\beta}\left(\mathbf{r}_{2}\right)+\psi_{\alpha}\left(\mathbf{r}_{2}\right) \cdot \psi_{\beta}\left(\mathbf{r}_{1}\right)\right], \\
& \psi_{A}\left(\mathbf{r}_{1}, \mathbf{r}_{2}\right)=\frac{1}{\sqrt{2}}\left[\psi_{\alpha}\left(\mathbf{r}_{1}\right) \psi_{\beta}\left(\mathbf{r}_{2}\right)-\psi_{\alpha}\left(\mathbf{r}_{2}\right) \psi_{\beta}\left(\mathbf{r}_{1}\right)\right],
\end{aligned}
$$

respectively. The anti-symmetric case can be conveniently written in matrix format as $\psi_{A}\left(\mathbf{r}_{1}, \mathbf{r}_{2}\right)=$ $\frac{1}{\sqrt{2}} \operatorname{det}\left[\begin{array}{ll}\psi_{\alpha}\left(\mathbf{r}_{1}\right) & \psi_{\alpha}\left(\mathbf{r}_{2}\right) \\ \psi_{\beta}\left(\mathbf{r}_{1}\right) & \psi_{\beta}\left(\mathbf{r}_{2}\right)\end{array}\right]$

A comparable concept in the scope of wavelets, also characterized by wave functions, would be the combination of different spatial wavelets 10 .

\subsection{Orbital Wavelets}

\subsubsection{Symmetric Case: Standard Wavelets}

Usual wavelet image analysis combines one-dimensional (1D) wavelets to generate a two-dimensional (2D) wavelet 1 , 44. This can be done by considering a scaling function $\varphi$ and a wavelet function $\psi$, a version for each dimension, abscissa and ordinate [8]. Thus, we have a $2 \mathrm{D}$ scale function $\varphi_{\mathrm{LL}}(x, y)=\varphi(x) \cdot \varphi(y)$ and three $2 \mathrm{D}$ wavelet functions:

$$
\begin{aligned}
\psi_{\mathrm{LH}}(x, y) & =\varphi(x) \cdot \psi(y), \\
\psi_{\mathrm{HL}}(x, y) & =\psi(x) \cdot \varphi(y), \\
\psi_{\mathrm{HH}}(x, y) & =\psi(x) \cdot \psi(y) .
\end{aligned}
$$

The wavelets $\psi_{\mathrm{LH}}(x, y)$ and $\psi_{\mathrm{HL}}(x, y)$ naturally exhibit reflection symmetry with respect to the plane $x=y$, i.e. $\psi_{\mathrm{LH}}(x, y)=\psi_{\mathrm{LH}}(y, x)$ and $\psi_{\mathrm{HL}}(x, y)=\psi_{\mathrm{HL}}(y, x)$. Such reflection symmetry is analogous to the symmetric wave function described in (1).

\subsubsection{Anti-symmetric Case: Orbital Wavelets}

Following this analogy, considering a single orthogonal wavelet system, we are compelled to pursue the definition of wavelets that could be regarded as the counterparts of the anti-symmetric wave function described in (2). Thus the combination of $\varphi(\cdot)$ and $\psi(\cdot)$ should be arranged to provide anti-symmetry, i.e. the $\hat{\psi}_{\mathrm{LH}}(x, y)$ and $\hat{\psi}_{\mathrm{HL}}(x, y)$ wavelets 


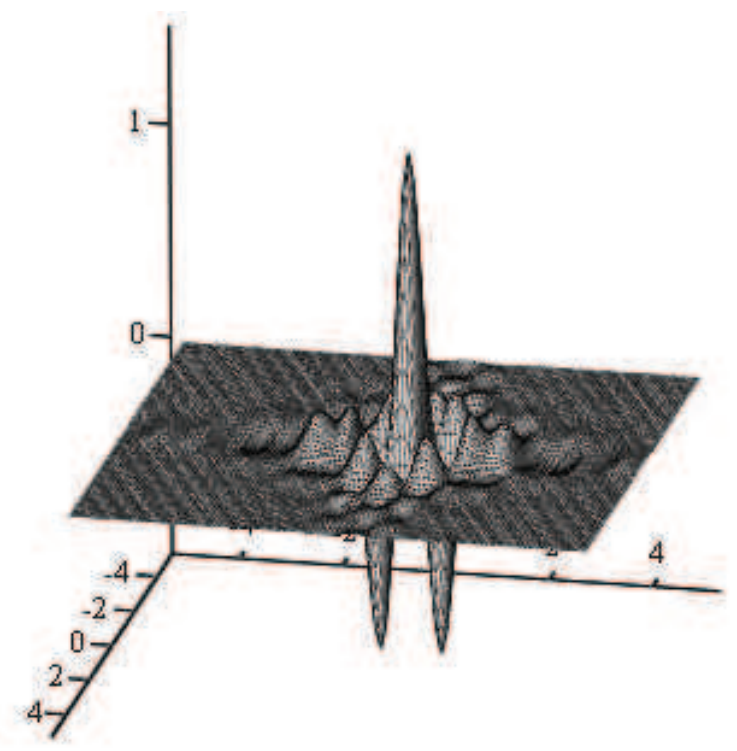

(a) $\psi_{\mathrm{LH}}(x, y)$

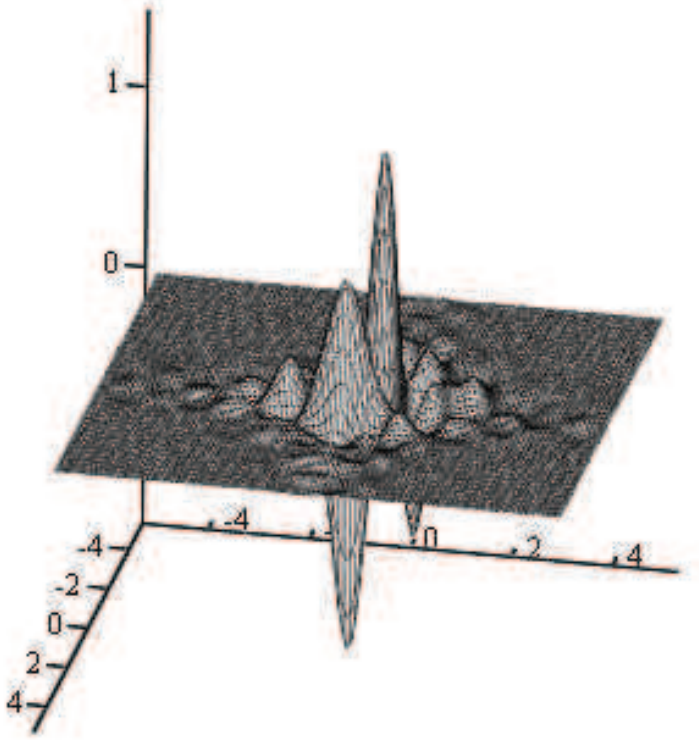

(b) $\hat{\psi}_{\mathrm{LH}}(x, y)$

Figure 1: 2D-Meyer decomposing functions of the secondary diagonal: (a) $\psi_{\mathrm{LH}}(x, y)$; (b) $\hat{\psi}_{\mathrm{LH}}(x, y)$.

should be such that:

$$
\begin{aligned}
& \hat{\psi}_{\mathrm{LH}}(x, y)=-\hat{\psi}_{\mathrm{LH}}(x, y) \\
& \hat{\psi}_{\mathrm{HL}}(x, y)=-\hat{\psi}_{\mathrm{HL}}(x, y)
\end{aligned}
$$

A solution to the above requirement is to define an orbital-inspired combination (cf. (2)) of $\varphi$ and $\psi$ according to the following:

$$
\hat{\psi}_{\mathrm{LH}}(x, y) \triangleq \frac{1}{\sqrt{2}}[\varphi(x) \cdot \psi(y)-\psi(x) \cdot \varphi(y)]
$$

In order to illustrate the effect of this definition we consider the case of the Meyer orthogonal wavelet [26]. Figure 1 shows the surface plots for the standard of the discussed functions.

Analogously, we define the wavelets related to the LL and HH decompositions according to the following expressions:

$$
\begin{gathered}
\hat{\varphi}_{\mathrm{LL}}(x, y) \triangleq \frac{1}{\sqrt{2}}\left[\varphi^{*}(x) \cdot \varphi(y)+\varphi^{*}(y) \cdot \varphi(x)\right], \\
\hat{\psi}_{\mathrm{HH}}(x, y) \triangleq \frac{1}{\sqrt{2}}\left[\psi^{*}(x) \cdot \psi(y)-\psi^{*}(y) \cdot \psi(x)\right] .
\end{gathered}
$$

The above definitions allows the analysis of images using continuous complex wavelets [21]. For real-valued wavelets, the above expressions collapse to the usual forms $\varphi(x) \cdot \varphi(y)$ or $\psi(x) \cdot \psi(y)$ present in standard wavelet analysis. Thus in the real case, although the proposed wavelet $\hat{\varphi}_{\mathrm{LL}}(x, y)$ in (7) is well-defined, the wavelet $\hat{\psi}_{\mathrm{HH}}(x, y)$ would collapse to zero.

An approach to address such degeneracy is to consider daughter wavelets at different scales. Therefore let us consider the 1D orthogonal [22,24] wavelet mother $\psi(x)$ equipped equipped with her daughter wavelets $\left\{\psi_{a, b}(x)\right\}_{a \neq 0, b \in R}$. 
The formalism shown in (8) can be extended by considering the inclusion of two wavelets $\psi_{a_{1}, b}(\cdot)$ and $\psi_{a_{2}, b}(\cdot)$ resulting in the following definition.

Definition 1 The function $2 D$-orbital at the scales $\left\{a_{1}, a_{2}\right\}$ is defined by:

$$
\hat{\psi}_{H H}(x, y) \triangleq \frac{1}{\sqrt{2}} \operatorname{det}\left[\begin{array}{ll}
\psi_{a_{1}, b}^{*}(x) & \psi_{a_{1}, b}^{*}(y) \\
\psi_{a_{2}, b}(x) & \psi_{a_{2}, b}(y)
\end{array}\right]
$$

which can be rewritten as:

$$
\begin{aligned}
\hat{\psi}_{H H}(x, y)= & \frac{1}{\sqrt{2\left|a_{1}\right|\left|a_{2}\right|}} \psi^{*}\left(\frac{x-b}{a_{1}}\right) \cdot \psi\left(\frac{y-b}{a_{2}}\right) \\
& -\frac{1}{\sqrt{2\left|a_{1}\right|\left|a_{2}\right|}} \psi\left(\frac{x-b}{a_{2}}\right) \cdot \psi^{*}\left(\frac{y-b}{a_{1}}\right) .
\end{aligned}
$$

The condition $a_{1} \neq a_{2}$ eliminates the degeneration $\hat{\psi}_{\mathrm{HH}}(x, y)=0$. This is to some extent in connection to the Pauli Exclusion Principle 13, which states that with a single-valued many-particle wave function is equivalent to requiring the wave function to be antisymmetric. An antisymmetric two-particle state is represented as a sum of states in which one particle is in state $\alpha$ and the other in state $\beta$. Besides, the relationship $\hat{\psi}_{\mathrm{HH}}(y, x)=-\hat{\psi}_{\mathrm{HH}}(x, y)$ ensures the desired asymmetry. Here, we use the same wavelet-mother, but at different scales. The 2D decomposition stated in Definition 1 results in a strict 2D wavelet.

\subsection{Mathematical Properties}

Proposition 1 The previously defined 2D-orbital function has oscillatory behavior satisfying the following properties:

(i) $\int_{-\infty}^{\infty} \hat{\psi}_{H H}(x, y) \mathrm{d} x=0$

(ii) $\int_{-\infty}^{\infty} \hat{\psi}_{H H}(x, y) \mathrm{d} y=0$,

(iii) $\int_{-\infty}^{\infty} \int_{-\infty}^{\infty} \hat{\psi}_{H H}(x, y) \mathrm{d} x \mathrm{~d} y=0$.

Proof: It follows that

$$
\int_{-\infty}^{\infty} \hat{\psi}_{\mathrm{HH}}(x, y) \mathrm{d} x=\frac{1}{\sqrt{2}} \psi_{a_{2}, b}(y) \cdot \overline{\psi_{a_{1}, b}^{*}(x)}-\frac{1}{\sqrt{2}} \overline{\psi_{a_{2}, b}(x)} \cdot \psi_{a_{1}, b}^{*}(y),
$$

where

$$
\overline{\psi_{a, b}(x)} \triangleq \int_{-\infty}^{\infty} \psi_{a, b}(x) \mathrm{d} x
$$

Therefore, the property (i) derives from the fact that $\psi_{a, b}(x), a=\left\{a_{1}, a_{2}\right\}$ be individual wavelets. The demonstration for property (ii) is similar, considering that

$$
\int_{-\infty}^{\infty} \hat{\psi}_{\mathrm{HH}}(x, y) \mathrm{d} y=\frac{1}{\sqrt{2}} \overline{\psi_{a_{2}, b}(y)} \cdot \psi_{a_{1}, b}^{*}(x)-\frac{1}{\sqrt{2}} \psi_{a_{2}, b}(x) \cdot \overline{\psi_{a_{1}, b}^{*}(y)} .
$$

The condition $\int_{-\infty}^{\infty} \int_{-\infty}^{\infty} \hat{\psi}_{\mathrm{HH}}(x, y) \mathrm{d} x \mathrm{~d} y=0$ follows from Fubini's theorem [34], regardless of the order of integration. 
Hereafter we assume an orthogonal wavelet system with unitary energy. In other words, the following conditions hold true:

(i) the inner product $\left\langle\psi_{a_{1}, b}, \psi_{a_{2}, b}\right\rangle=0$, i.e., the following integrals cancel out $\forall a_{1} \neq a_{2}$ :

$$
\int_{-\infty}^{\infty} \psi_{a_{1}, b}(x) \cdot \psi_{a_{2}, b}^{*}(x) \mathrm{d} x=\int_{-\infty}^{\infty} \psi_{a_{1}, b}^{*}(x) \cdot \psi_{a_{2}, b}(x) \mathrm{d} x=0,
$$

and

(ii) $\int_{-\infty}^{\infty}\left|\psi_{a_{1}, b}(x)\right|^{2} \mathrm{~d} x=1$.

It is also noteworthy that

$$
\left\langle\psi_{a_{1}, b}, \psi_{a_{2}, b}\right\rangle^{*}=\left\langle\psi_{a_{2}, b}, \psi_{a_{1}, b}\right\rangle
$$

Proposition 2 The 2D-orbital functions have normalized energy.

Proof: Expanding the expression $\left|\hat{\psi}_{\mathrm{HH}}(x, y)\right|^{2}=\hat{\psi}_{\mathrm{HH}}(x, y) \cdot \hat{\psi}_{\mathrm{HH}}^{*}(x, y)$ yields cross-product terms in the following form

$$
\operatorname{cross}(x, y) \triangleq-\psi_{a_{1}, b}(x) \cdot \psi_{a_{2}, b}^{*}(y) \cdot \psi_{a_{1}, b}(y) \cdot \psi_{a_{2}, b}^{*}(y)
$$

and its complex conjugate $\operatorname{cross}^{*}(x, y)$. Performing the integration with respect to $x$ and $y$ yields:

$$
\int_{-\infty}^{\infty} \operatorname{cross}(x, y) \mathrm{d} x=-2 \cdot \psi_{a_{2}, b}^{*}(y) \psi_{a_{1}, b}(y) \cdot \int_{-\infty}^{\infty} \psi_{a_{1}, b}^{*}(x) \psi_{a_{2}, b}(x) \mathrm{d} x
$$

and

$$
\int_{-\infty}^{\infty} \operatorname{cross}(x, y) \mathrm{d} y=-2 \cdot \psi_{a_{2}, b}^{*}(x) \psi_{a_{1}, b}(x) \cdot \int_{-\infty}^{\infty} \psi_{a_{1}, b}^{*}(y) \psi_{a_{2}, b}(y) \mathrm{d} x
$$

Invoking the orthogonality condition we obtain that all cross terms are void. The remaining possibly nonnull terms are:

$$
\left|\hat{\psi}_{\mathrm{HH}}(x, y)\right|^{2}=\frac{1}{2}\left|\psi_{a_{1}, b}(x)\right|^{2} \cdot\left|\psi_{a_{2}, b}(y)\right|^{2} \cdot\left|\psi_{a_{1}, b}(y)\right|^{2} \cdot\left|\psi_{a_{2}, b}(x)\right|^{2}
$$

and therefore, because of the normalized energy condition, we have:

$$
\int_{-\infty}^{\infty} \int_{-\infty}^{\infty}\left|\hat{\psi}_{\mathrm{HH}}(x, y)\right|^{2} \mathrm{~d} x \mathrm{~d} y=1
$$

It is possible (more easily) to combine orthogonal 1D-wavelets and use them to build a new 2D-wavelet.

Proposition 3 The 2D-orbital function is a $2 D$ wavelet.

Proof: Let $\Psi(\omega)$ and $\Psi_{a, b}(\omega)$ be the Fourier transforms of the wavelet $\psi(t)$ and the daughter wavelets $\psi_{a, b}(t)$, respectively. If the admissibility condition holds [6],

$$
\int_{-\infty}^{\infty} \frac{|\Psi(\omega)|^{2}}{|\omega|} \mathrm{d} \omega<\infty
$$




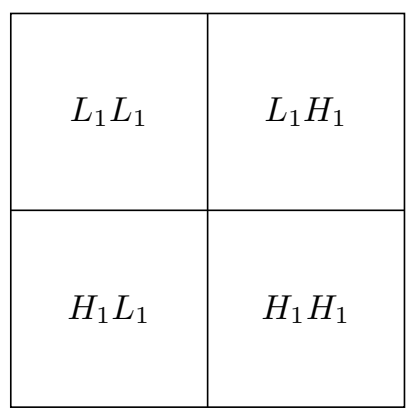

(a) First leve

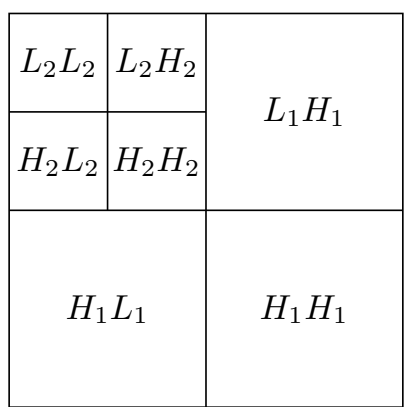

(b) Second level

Figure 2: Image decomposition scheme according to the standard 2D wavelet decomposition.

then $\int_{-\infty}^{\infty} \frac{\left|\Psi_{a, b}(\omega)\right|^{2}}{|\omega|} \mathrm{d} \omega<\infty$, since $\Psi_{a, b}(\omega)=\sqrt{|a|} \Psi(a \omega) e^{-j \omega b}$ 25]. Let us now evaluate the condition for the 2D case. If the Fourier transform pair $\hat{\psi}_{\mathrm{HH}}(x, y) \leftrightarrow \hat{\Psi}_{\mathrm{HH}}(u, v)$ does exist, then the $2 \mathrm{D}$ spectrum of $\hat{\psi}_{\mathrm{HH}}$ can be computed in terms of the Fourier spectrum of $\psi$ :

$$
\hat{\Psi}_{\mathrm{HH}}(u, v)=\frac{\sqrt{\left|a_{1} a_{2}\right|}}{\sqrt{2}}\left[\Psi\left(a_{1} u\right) \Psi^{*}\left(a_{2} v\right)-\Psi\left(a_{2} u\right) \Psi^{*}\left(a_{1} v\right)\right]
$$

From the generalized Parseval-Plancherel energy theorem 8, 34, the cross-terms vanish due to the orthogonality. Thus, we have

$$
\left|\hat{\Psi}_{\mathrm{HH}}(u, v)\right|^{2}=\frac{\left|a_{1} a_{2}\right|}{2}\left|\Psi\left(a_{1} u\right)\right|^{2} \cdot\left|\Psi\left(a_{2} v\right)\right|^{2}+\frac{\left|a_{1} a_{2}\right|}{2}\left|\Psi\left(a_{2} u\right)\right|^{2} \cdot\left|\Psi\left(a_{1} v\right)\right|^{2} .
$$

Then, from the marginal admission conditions of the $1 \mathrm{D}$ daughter wavelets, we have that

$$
\int_{-\infty}^{\infty} \int_{-\infty}^{\infty} \frac{\left|\hat{\Psi}_{\mathrm{HH}}(u, v)\right|^{2}}{|u| \cdot|v|} \mathrm{d} u \mathrm{~d} v<\infty .
$$

\section{Orbital Wavelet-based Image Decomposition}

The standard 2D wavelet decomposition effects coefficient matrices representing vertical, horizontal, and diagonal structures denoted by sub-images $\left\{L_{1} L_{1}, L_{1} H_{1}, H_{1} L_{1}, H_{1} H_{1}\right\}$ and $\left\{L_{2} L_{2}, L_{2} H_{2}, H_{2} L_{2}, H_{2} H_{2}\right\}$ for the first and second level decompositions [40]. Figure 2 illustrate the structures. Noticed that the terms $L_{1} L_{1}, L_{2} L_{2}, H_{1} H_{1}$, and $H_{2} H_{2}$ on the main diagonal correspond to a part of the standard wavelet decomposition of the image into two levels. The proposed wavelet analysis results in a similar structure with sub-images $\left\{\widehat{L_{1} L_{1}}, \widehat{L_{1} H_{1}}, \widehat{H_{1} L_{1}}, \widehat{H_{1} H_{1}}\right\}$ and $\left\{\widehat{L_{2} L_{2}}, \widehat{L_{2} H_{2}}, \widehat{H_{2} L_{2}}, \widehat{H_{2} H_{2}}\right\}$ in a two-level decomposition. Despite the similarity, the sub-images are computed according to the orbital wavelets. The resulting scheme is depicted in Figure 3 .

Considered the symlet wavelet of order 4 (symlet4) 9, we applied the proposed decomposition to the standard image woman [27; the resulting sub-images are shown in Figure 4 . For a qualitative comparison, we included the sub-images obtained from the usual wavelet decomposition. The computation was performed in the Matlab environement 27. It is worth nothing that the subtraction of the images resulting from $\psi_{\mathrm{LH}}(x, y)$ and $\psi_{\mathrm{HL}}(x, y)$ results in the image obtained by the wave function in (6). 


\begin{tabular}{|l|l|}
\hline${\widehat{L_{1} L_{1}}}$ & ${\widehat{L_{1} H_{1}}}$ \\
${\widehat{H_{1} L_{1}}}$ & $\widehat{H_{1} H_{1}}$ \\
\hline
\end{tabular}

(a) First level

\begin{tabular}{|c|c|c|}
\hline${\widehat{L_{2} L_{2}}}$ & ${\widehat{L_{2} H_{2}}}_{2}$ & \\
\hdashline${\widehat{H_{2} L_{2}}}_{2}$ & ${\widehat{H_{2} H_{2}}}_{1}$ & \\
& ${\widehat{H_{1} L_{1}}}$ & \\
& ${\widehat{H_{1} H_{1}}}$ \\
\hline
\end{tabular}

(b) Second level

Figure 3: Image decomposition scheme according to the $2 \mathrm{D}$ orbital wavelet decomposition. A two-level wavelet-orbital decomposition.
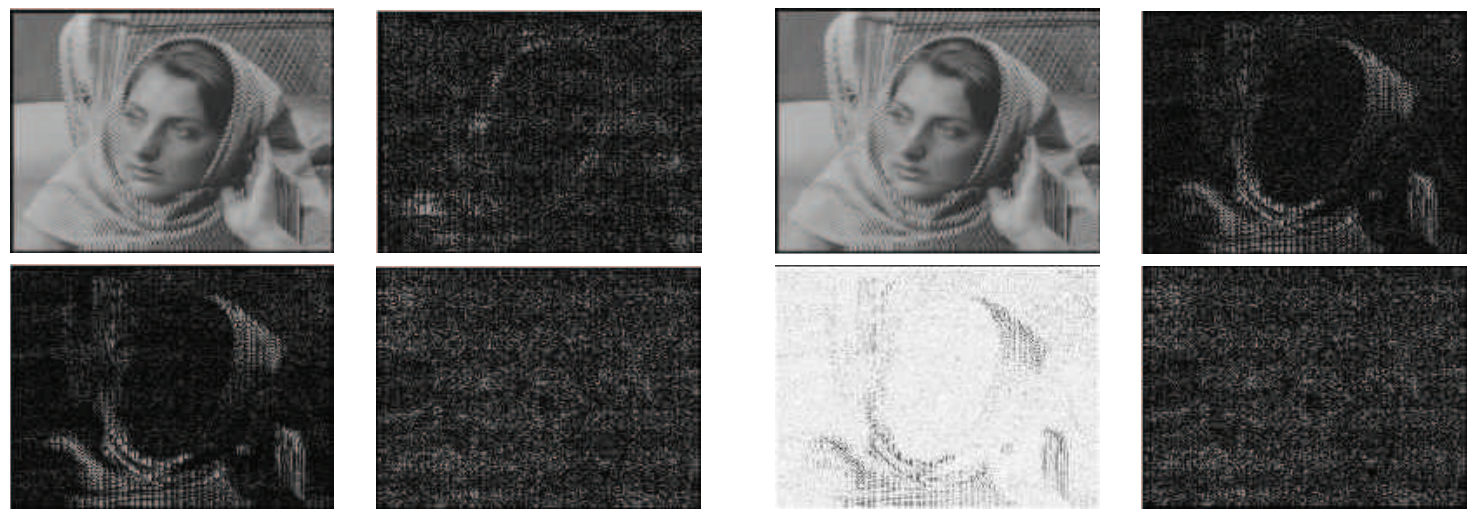

(a) standard wavelet decomposition

(b) Proposed decomposition

Figure 4: First-level decomposition woman image using symlet4 wavelet as defined in Matlab according to (a) the standard wavelet decomposition and (b) the proposed decomposition. 


\section{Concluding Remarks and Future Work}

This paper offers an alternative approach for image decomposition engendered by asymmetric orthogonal wavelets whose definition is inspired by the wave function theory from particle physics. Despite the focus being essentially on still image analysis, the proposed approach allows a fully scalable multimedia decomposition. It remains to be investigated the potential of this methodology for image compressing [12, in 3D processing and scalable coding for multimedia schemes [30]. Applications in other scenarios such as wavelet-based watermarking [16] or steganography [32 also deserve an investigation. To the best of our knowledge, this is the first work to link the exchanging formalism from particle wave functions to wavelets analysis.

\section{Acknowledgments}

The second and third authors acknowledge the partial support from the Brazilian agencies CAPES and CNPq, respectively.

\section{References}

[1] M. Antonini, M. Barlaud, P. Mathieu, and I. Daubechies, Image coding using wavelet transform, IEEE Transactions on Image Processing 1 (1992), 205-220. 10

[2] J. Ashmead, Morlet wavelets in quantum mechanics, Quanta 1 (2012), 58-70. 11

[3] J. F. Aujol, G. Gilboa, T. Chan, and S. Ocher, Structure-texture image decomposition: modeling, algorithms, and parameter selection, International Journal of Computer Vision 67 (2006), 111-136. 1

[4] A. Baganne, I. Bennour, M. Elmarzougui, R. Gaiech, and E. Martin, A multi-level design flow for incorporating IP cores: Case study of $1 D$ wavelet IP integration, IEEE Design, Automation and Test in Europe Conference and Exhibition 1 (2003), 250-255. 11

[5] A. Beiser, Concepts of modern physics, McGraw-Hill, 1994. 12

[6] A. Boggess and F. J. Narcowich, A first course in wavelets with fourier analysis, Wiley, 2009. 15

[7] V. Bottreau, M. Bnetire, and B. Felts, A fully scalable 3D subband video codec, IEEE International Conference on Image Processing 2 (2001), 1017-1020. 1

[8] C. S. Burrus, R. A. Gopinath, and H. Guo, Introduction to wavelets and the wavelet transform - A primer, Prentice-Hall, 1998. 11,56

[9] I. Daubechies, Ten lectures on wavelets, Society for Industrial and Applied Mathematics, 1992.

[10] H. M. de Oliveira, Análise de sinais para engenheiros: Uma abordagem via wavelets, Editora Brasport, 2007.12

[11] M. Van der Schaar and D. S. Turaga, Multiple description scalable coding using wavelet-based motion compensated temporal filtering, Proceedings of IEEE International Conference on Image Processing 3 (2003), 489-492. 11

[12] R. DeVore, B. Jawwerth, and B. Lucier, Image compression through wavelet transform coding, IEEE Trans. on Information Theory 38 (1995), 719-746. 118

[13] I. Duck and E. C. G. Sudarshan, Pauli and the spin-statistics theorem, World Scientific, 1998. 124

[14] R. M. Eisberg, Fundamentals of modern physics, J. Wiley \& Sons, 2007. 2

[15] M. L. Hilton, B. D. Jawerth, and A. Sengupta, Compressing still and moving images with wavelets, Multimedia Systems 2 (1994), 218-227. $1[1$

[16] J. Hu, J. Huang, D. Huang, and Y. Q Shi, Image fragile watermarking based on fusion of multi-resolution tamper detection, Electronics Letters 38 (2002), 1512-1513. 18

[17] C. E. Jacobs, A. Finkelstein, and D. H. Salesin, Fast multiresolution image querying, Proceeding of the Special Interest Group on Graphics and Interactive Techniques 1 (1995), 277-286. 11

[18] M. Jallouli, W. B. H. Khélifa, A. Ben Mabrouk, and M. A. Mahjoub, Toward recursive spherical harmonics issued bi-filters: Part II: an associated spherical harmonics entropy for optimal modeling, Soft Computing (August 2019). 11

[19] M. Jallouli, M. Zemni, A. Ben Mabrouk, and M. A. Mahjoub, Toward recursive spherical harmonics-issued bi-filters: Part I: theoretical framework, Soft Computing 23 (October 2019), no. 20, 10415-10428. 1

[20] B. G. Kim, J. I. Shim, and D. J. Park, Fast image segmentation based on multi-resolution analysis and wavelets, Pattern Recognition Letters 24 (2003), 2995-3006. 1

[21] N. Kingsbury, Image processing with complex wavelets, Phil. Transactions of Royal Society London, A Mathematical, Physical and Engineering Sciences 357 (1999), 2543-2560. 13

[22] W. M. Lawton, Necessary and sufficient conditions for constructing orthonormal wavelet bases, Journal of Mathematic Physics 32 (1991), 57-61. 3

[23] J. S. Lim, Two-dimensional signal and image processing, Prentice Hall, 1990. 
[24] S. Maaß, Families of orthogonal 2D wavelets, Society for Industrial and Applied Mathematics - Journal of Applied Analysis 27 (1996), 1454-1481. 13

[25] S. G. Mallat, A wavelet tour of signal processing, 2nd ed., Academic Press, 1999. 116

[26] Y. Meyer, Ondelettes et opérateurs, Hermann, 1990.

[27] M. Misiti, Y. Misiti, G. Oppenheim, and J. M. Poggi, Wavelet toolbox user's guide 4, 2nd ed., The MathWorks, 2002. 16

[28] S. Muraki, Volume data and wavelet transforms, IEEE Computer Graphics and Applications 13 (1993), 50-56.

[29] O. Nicolis and J. Mateu, 2D anisotropic wavelet entropy with an application to earthquakes in Chile, Entropy $\mathbf{1 7}$ (2015), no. 6, 4155-4172. 1

[30] J. R. Ohm, M. Van der Schaar, and J. W. Woods, Interframe wavelet coding: motion picture representation for universal scalability, Image Communication Signal Processing 19 (2004), 877-908. 11 8

[31] H. M. Radha, M. Van der Schaar, and Y. Chen, The MPEG-4 fine-grained scalable video coding method for multimedia streaming over IP, IEEE Trans. on Multimedia 3 (2001), 53-58. 1

[32] S. Rekik, D. Guerchi, H. Hamam, and S. A. Selouani, Audio steganography coding using the discrete wavelet transforms, International Journal of Computer Science and Security 6 (2012), 79-93. 18

[33] Jos B. T. M. Roerdink, Jos B. T. M, Roerdink, and M. A. Westenberg, Wavelet - based volume visualization, Nieuw Archief voor Wiskunde 17 (1999), 149-158. 1

[34] W. Rudin, Principles of mathematical analysis, Vol. 62, McGraw-Hill, 1976. 14] 6]

[35] S. Saha, Image compression - from DCT to wavelets: a review, Crossroads 6 (2000), 12-21. 11

[36] P. Schröder, Wavelets in computer graphics, Proceedings of the IEEE 84 (1996), 615-625. 11

[37] A. Skodras, C. Chistopoulos, and T. Ebrahimi, The JPEG 2000 still image compression standard, IEEE Signal Processing Mag. 18 (2001), 36-58. 11

[38] I. Sodagar and Y. Q. Zhang, Scalable picture coding for multimedia applications, Wavelet, subband and block transforms in communication and multimedia 504 (2002), 295-322. 11

[39] E. J. Stollnitz and T. D. Derose, Wavelets for computer graphics, Morgan-Kaufmann Pub, 1996.11

[40] Gilbert Strang and Truong Nguyen, Wavelets and filter banks, Wellesley-Cambridge Press, 1997. 16

[41] D. M. Sullivan, Quantum mechanics for electrical engineers, IEEE Series on Microelectronics Systems, Wiley, 2003.

[42] D. Taubman and A. Zakhor, Multirate 3-D subband coding of video, IEEE Trans. on Image Processing 3 (1994), 572-588. 11

[43] B. E. Usevitch, A tutorial on modern lossy wavelet image compression: foundations of JPEG 2000, IEEE Signal Proc. Mag. 18 (2001), 22-35. 1

[44] M. Vetterli and J. Kovacevic, Wavelets and subband coding, Prentice-Hall, 1990. 11 2

[45] M. Zemni, M. Jallouli, A. Ben Mabrouk, and M. Ali Mahjoub, Explicit Haar-Schauder multiwavelet filters and algorithms. part II: Relative entropy-based estimation for optimal modeling of biomedical signals, International Journal of Wavelets, Multiresolution and Information Processing 17 (2019), no. 4, 1950038-1-1950038-25. 1 